\title{
Implementación de un programa de estimulación multisensorial en personas mayores con trastorno neurocognitivo
}

\author{
Implementation of a multisensory stimulation \\ program in elderly people with neurocognitive \\ disorders
}

García-Pérez, Mariana Monserrat; Morales-Hernández, Diego; Ramírez-Becerra, Brenda Anahí; Ugalde-Chávez, María de Lourdes; Vargas-Ortuño, Sharon Alinthia; Hernández-Valle, Verónica

\section{Mariana Monserrat García-Pérez \\ Universidad Autónoma de Querétaro, México \\ Diego Morales-Hernández \\ Universidad Autónoma de Querétar, México \\ Brenda Anahí Ramírez-Becerra \\ Universidad Autónoma de Querétaro, México \\ María de Lourdes Ugalde-Chávez \\ Universidad Autónoma de Querétaro, México \\ Sharon Alinthia Vargas-Ortuño \\ Universidad Autónoma de Querétaro, México \\ Verónica Hernández-Valle \\ veronica.hernandez@uaq.mx \\ Universidad Autónoma de Querétaro, México}

\section{Lux Médica}

Universidad Autónoma de Aguascalientes, México

ISSN: 2007-1655

Periodicidad: Cuatrimestral

vol. 16, núm. 48, 2021

luxmedica.editorial@gmail.com

Recepción: 08 Febrero 2021

Aprobación: 24 Julio 2021

URL:

https://revistas.uaa.mx/index.php/luxmedica/article/view/3273

Poítica de acceso abierto La Revista Lux Médica proporciona un acceso abierto a su contenido, basado en el principio de que ofrecer un acceso libre a las investigaciones ayuda a incrementar el intercambio global del conocimiento. La LM no cobra ni cobrará ningún cargo a sus lectores por concepto de suscripción, ni a los autores por enviar, procesar o publicar sus artículos. Como condición de publicación, los autores acuerdan liberar sus derechos de autor bajo una licencia compartida, específicamente la licencia de Creative Commons Reconocimiento-NoComercial-Compartir Igual 4.0 Internacional Esta licencia permite a cualquier persona compartir, copiar y redistribuir el material en cualquier medio o formato bajo
Resumen: Introducción Se estima que alrededor del mundo hay más de 50 millones de personas que presentan algún trastorno neurocognitivo. Actualmente no se dispone de un tratamiento que pueda curar o revertir la evolución progresiva de sus síntomas psicológicos y de comportamiento. Por ello, es de vital importancia la aplicación de una intervención que posibilite un estado de alteración leve o moderado y que prevenga la evolución de la enfermedad. Objetivo: Implementación de un programa de estimulación multisensorial en personas mayores con trastorno neurocognitivo menor y mayor, para la prevención y manejo de alteraciones conductuales, y reducción del grado de distrés experimentado por el cuidador primario. Metodología: Estudio cuasi-experimental, longitudinal, analítico comparativo y retrospectivo durante el periodo julio-diciembre de 2019. Incluyó un total de 20 personas mayores de 65 años con diagnóstico de trastorno neurocognitivo. Se utilizaron para la evaluación: Global Deterioration Scale (GDS) y el Neuropsychiatric Inventory (NPI), además se creó un "Perfil sensorial" a cada usuario. La estimulación multisensorial se acompañó de ejercicio físico y actividades motrices. Resultados: Se encontró una diferencia estadísticamente significativa entre la primera y la segunda evaluación. En las alteraciones conductuales de los usuarios con un valor de $\mathrm{z}=-3.7,(\mathrm{p}=0.00)$ y referente al distrés del cuidador con un valor de $\mathrm{z}=-2.5,(\mathrm{p}=0.01)$. Conclusión: Se mostró un efecto positivo sobre la situación clínica general que experimentaba el usuario; disminuyendo las alteraciones conductuales y el distrés del cuidador primario, así como facilitando la intervención fisioterapéutica.

Palabras clave: estimulación multisensorial, trastorno neurocognitivo, alteraciones conductuales.

Abstract: Introduction: Approximately more than 50 million people around the world lives with a neurocognitive disorder. Nowadays, there is no treatment available to heal or reverse the progressive disease evolution, psychological and behavioral symptoms. Therefore, it is vital to apply an intervention that 
los siguientes términos: - Dar crédito al autor del texto - No hace uso del material con propósitos comerciales - No transformar o modificar el material. Los autores ceden el derecho de la primera publicación a esta revista, pero conservarn sus derechos de autor, de tal forma que pueden realizar otros acuerdos contractuales independientes y adicionales para la distribución no exclusiva de la versión del artículo publicado en esta revista ( por ejemplo, incluirlo en un repositorio institucional o publicarlo en un libro) siempre que indiquen claramente que el trabajo se publicó por primera vez en ésta.

\section{c) (i) (2)}

Esta obra está bajo una Licencia Creative Commons AtribuciónNoComercial-CompartirIgual 4.0 Internacional.

Cómo citar este artículo: Hernández Valle, V., Ramírez Becerra, B. A., Ugalde Chávez, M. de L., Vargas Ortuño, S. A., Morales Hernández, D., García Pérez, M. M. Implementación de un programa de estimulación multisensorial en personas mayores con trastorno neurocognitivo. Lux Médica, 16(48). Recuperado a partir de https://revistas.uaa.mx/index.php/luxmedica/article/view/3273 enables a mild or moderate disturbance condition and supports the prevention of the evolution of the disease. Objective: Implement a multisensory stimulation program in elderly people with a neurocognitive disorder to prevent and manage behavioral disorders and reduce the degree of distress experienced by the primary caregiver. Methods: Quasi-experimental, longitudinal, comparative analytical, and retrospective study during the period July-December 2019. The study included 20 people over 65 years old with a diagnosis of neurocognitive disorder. For the evaluation were used: The Global Deterioration Scale (GDS) and the Neuropsychiatric Inventory (NPI), also a "Sensory Profile" format was generated for each user. Physical exercise and motor activities were considered into the multisensory stimulation. Results: A statistically significant difference was found between the first and the second evaluation related to user behavioral disorders with a value of $\mathrm{z}=-3.7(\mathrm{p}=0.00)$ and concerning caregiver distress with a value of $z=-2.5(p=0.01)$. Conclusion: A positive effect on the general clinical situation experienced by the user was showed, reducing behavioral alterations and distress on the primary caregiver, additionally facilitating the physiotherapeutic intervention.

Keywords: multisensory stimulation, neurocognitive disorders, behavioral disturbances.

\section{INTRODUCCIÓN}

Con la llegada del envejecimiento se presentan cambios morfológicos, bioquímicos, metabólicos y circulatorios que, dependiendo del grado de funcionalidad previo y la plasticidad cerebral de cada individuo, pueden jugar un rol importante en la evolución a alteraciones cognitivas. ${ }^{1}$ Este proceso se manifiesta en la persona mayor sana como un descenso en la funcionalidad psicomotriz, que no interfiere con las actividades de la vida diaria. ${ }^{2}$

De acuerdo con el Manual diagnóstico y estadístico de las enfermedades mentales (DSM) de la American Psychiatric Association (APA) en su quinta versión (DSM-5), el concepto de "trastorno neurocognitivo", se divide en tres categorías: delirium, trastorno neurocognitivo menor para referirse a deterioro cognitivo leve y trastorno neurocognitivo mayor, conocido como demencia. ${ }^{3}$

El trastorno neurocognitivo menor se define como aquel deterioro que supera lo normalmente esperable para la edad, pero que no cumple con criterios de demencia, pues la funcionalidad está aún preservada; mientras que el trastorno neurocognitivo mayor es definido como un síndrome clínico causado por neurodegeneración y se caracteriza por el deterioro progresivo de las capacidades cognitivas, impactando directamente en la funcionalidad y en la independencia de quien la padece. Básicamente, se diferencian en función de la intensidad de los síntomas y su repercusión en la funcionalidad del paciente. ${ }^{3}$

Estudios realizados en 2010 han encontrado que aquellos sujetos de mayor edad que tienen bajo rendimiento cognitivo son más propensos a desarrollar demencia tipo Alzheimer en comparación con quienes presentan un rendimiento cognitivo alto. ${ }^{4}$ Según el Informe Mundial sobre el Alzheimer, se estima que hay 
más de 50 millones de personas que viven con demencia alrededor del mundo, lo cual podría aumentar a 152 millones para el año 2050, principalmente debido a un incremento en la sobrevida. Por lo que, si ahora hay casi 350,000 personas que padecen algún tipo de trastorno neurocognitivo en México, para el año 2025 este número podría rebasar el millón. ${ }^{5}$

Algunas de las principales manifestaciones conductuales y psicológicas de la enfermedad son la desconexión con el entorno, la apatía o indiferencia, ideas delirantes, alucinaciones, agitación y/o agresividad, depresión o disforia, ansiedad, euforia, desinhibición, irritabilidad e inestabilidad, conducta motora anómala, alteraciones del sueño y del apetito, así como desórdenes alimenticios. Diversos autores exponen cómo la aplicación de estímulos en un ambiente controlado disminuye o controla la manifestación de estas conductas. ${ }^{6}$ Además, se producen beneficios tales como relajación, disminución del estrés, un ambiente seguro para explayarse, mejora de la comunicación y las relaciones personales, mejor concentración e integración cognitiva, mayor interacción social y la posibilidad de trabajar las emociones; reduciendo el cansancio del cuidador primario y brindándole al personal sanitario la posibilidad de un mejor manejo del usuario. ${ }^{7}$

Los síntomas conductuales son importantes ya que tienen una gran influencia en la calidad de vida del enfermo y de sus familiares. Estos se identifican en el 70 al $90 \%$ de las personas que viven con algún trastorno neurocognitivo, siendo uno de los factores principales de institucionalización. ${ }^{8}$

En la actualidad, no se dispone de ningún tratamiento que pueda curar o revertir la evolución progresiva de los trastornos neurocognitivos, de los síntomas psicológicos y del comportamiento que acompañan a estos. Sin embargo, existen intervenciones que están siendo investigadas con la finalidad de impactar en la mejora de la calidad de vida, así como en la de sus cuidadores y familiares. ${ }^{9}$

En 2020, el Informe Mundial sobre el Alzheimer: diseño, dignidad, demencia; sugiere como una alternativa no farmacológica el diseño de entornos seguros para las personas con demencia, con la finalidad de mejorar la orientación y la movilidad. Enfatizando que no necesariamente deben ser costosos o difíciles de crear; al contrario, cuanto más sencillos sean los principios en los que se basa el diseño más fácil será su replicación en cualquier contexto. ${ }^{10}$

Uno de los métodos de intervención más destacados, que involucra la creación de espacios adecuados, es la estimulación multisensorial (EM), conocida como método Snoezelen, que, de acuerdo con el World Wide Snoezelen, fue creada en la década de los 70 por los terapeutas holandeses Jan Hulsegge y Ad Verheul en el centro de Hartenberg. El método no tiene una manera exacta de aplicarse, ya que debe ser dirigido a las necesidades de cada paciente; sin embargo, se proponen algunos principios que deben tomarse en cuenta al momento de realizar la intervención. El primero es que la atmósfera debe ser un medio facilitador para llevar a cabo la tarea; debe ser confortable y segura. Cada estímulo que se encuentre dentro del espacio asignado debe ser modulado. Otra de las consideraciones propuestas es el uso correcto del tiempo, el cual se refiere a proporcionar estímulos de forma gradual y con un orden establecido que evite sobreestimular al paciente. ${ }^{11}$ 
La EM puede llevarse a cabo dentro de salas adaptadas con diferentes instrumentos dirigidos a cada uno de los sentidos, o en un espacio abierto, siempre y cuando los principios expuestos se encuentren cubiertos. ${ }^{12}$ La EM se destaca como una alternativa terapéutica viable y de bajo costo.

Una metodología adecuada para la implementación de un programa de EM proporciona al personal de salud un gran complemento terapéutico en la atención del paciente con trastorno neurocognitivo, y facilita la replicación en cualquier centro dedicado a la atención de ellos. Para su correcta aplicación resulta primordial la identificación de aquellos individuos que pudieran beneficiarse y, posterior a ello, la creación de un espacio donde llevar a cabo la intervención. Un lugar donde el usuario se convierta en el protagonista de su aprendizaje y donde el fisioterapeuta sea un mediador durante el proceso. Este estudio tuvo como objetivo la implementación de un programa de EM en personas mayores con trastorno neurocognitivo, para la prevención y el manejo de alteraciones conductuales, así como la facilitación de un abordaje terapéutico óptimo que permita reducir el grado de distrés en el cuidador primario.

\section{MÉTODOS}

Se realizó un estudio cuasi-experimental, longitudinal, analítico comparativo y retrospectivo durante el periodo julio-diciembre del año 2019, con la finalidad de implementar un programa de EM; el cual incluyó un total de 20 personas mayores (PM) de 65 años pertenecientes a la Fundación Alzheimer I.A.P. de la Ciudad de Querétaro, Querétaro; con trastorno neurocognitivo, independientemente del género y edad, con asistencia mínima de tres veces a la semana y con consentimiento informado signado por familiar o persona física responsable. Aquellos cuya situación clínica incluyera ceguera, sordera, enfermedades agudas, hospitalización o que no aceptaran participar en el estudio fueron excluidos. Los criterios de eliminación fueron: deceso o baja de la institución.

La presente investigación se desarrolló de acuerdo a los principios establecidos en el código de Nuremberg y en cumplimiento con el Reglamento de la Ley General de Salud en Materia de Investigación para la Salud, Título II: De los Aspectos Éticos de la Investigación en Seres Humanos, Capítulo I; considerándose de riesgo mínimo, de acuerdo al artículo 17.

\section{Análisis estadistico}

La descripción de las variables cualitativas se realizó mediante distribución de frecuencias absolutas y relativas expresadas en porcentajes. El análisis de variables cuantitativas se realizó con medidas de tendencia central como el promedio y la mediana; de dispersión como rango y desviación estándar.

Para analizar los cambios en el grado de deterioro cognitivo (DC), la gravedad y frecuencia de las alteraciones conductuales del usuario, así como el distrés del cuidador posterior al programa de EM; se utilizó estadística paramétrica con el estadístico de prueba $t$-Student y estadística no paramétrica con la prueba de signo de Wilcoxon, donde para decidir sobre la pertinencia de aplicar estadística paramétrica o no, se evaluó la distribución normal de los datos con el estadístico 
Shapiro-Wilk. Los valores de significación alfa considerados como significativos fueron $<0.05$. El procesamiento de los datos y construcción de las gráficas se realizó con los programas Excel y SPSS V 20.

\section{Asignación de usuarios}

En primera instancia, se asignó un cuidador y un fisioterapeuta por cada cuatro usuarios; responsable de evaluar, recabar los resultados e intervenir durante la implementación del programa, el cual se distribuyó en el cronograma que se muestra en la Tabla 1.

Tabla 1. Cronograma de actividades multisensoriales

\begin{tabular}{|c|c|c|c|c|c|}
\hline Día de la semana & Lunes & Martes & Miércoles & Jueves & Viernes \\
\hline Sentido* & Vista y Oído & Tacto y Esquema Corporal & Vista y Olfato & Esquema Corporal y Oído & Tacto y Olfato \\
\hline \multicolumn{6}{|c|}{ Ejercicio físico/actividades motrices. } \\
\hline
\end{tabular}

\section{Evaluación}

La evaluación se realizó dos veces, al inicio y al final del programa de estimulación multisensorial. La primera tuvo como objetivo recabar datos generales por medio de una ficha de identificación y la aplicación de las escalas seleccionadas y, cuatro meses después, la segunda sirvió para identificar los cambios en el usuario y su cuidador tras la intervención multisensorial.

Para la evaluación se utilizaron dos herramientas. La Global Deterioration Scale (GDS), que tiene como objetivo principal evaluar el grado de DC por medio de los síntomas cognitivos y las alteraciones funcionales, se clasifica en siete estadios que se distinguen clínicamente y van desde la cognición normal a la demencia muy grave. ${ }^{13}$ El Neuropsychiatric Inventory (NPI) es un test de screening de las conductas más comunes de las demencias, cuyo objetivo es evaluar su gravedad y frecuencia, y el distrés producido en el cuidador. ${ }^{14}$

\section{Elaboración de perfil sensorial}

Se diseñó un formato denominado "Perfil sensorial" con la finalidad de establecer una descripción inicial de la interacción del usuario en las actividades sensoriales mediante observación directa, con el objetivo de evidenciar de forma cualitativa los cambios presentados durante el periodo de intervención. El detectar sus preferencias sensoriales permitió minimizar las alteraciones conductuales que pudieran suscitarse durante la terapia. 


\section{Habilitación del área}

Para dar inicio al programa de EM, se habilitó un área en la que se dispuso de material para trabajar cada sentido; con el objetivo de evocar recuerdos y manejar las alteraciones del comportamiento. Posteriormente, se realizaron protocolos de intervención para cada sentido que incluían objetivos, consideraciones previas, materiales, descripción del proceso y tiempo requerido para la ejecución. Cada sesión inició informando de manera clara y sencilla en qué consistiría la actividad, procurando la orientación en tiempo y espacio de forma continua.

La intervención se llevó a cabo con lo establecido en el cronograma. Cada sesión de EM tuvo una duración de 30 minutos, acompañada de 15 minutos de ejercicio físico y actividades motrices cuya demanda se extendió a desafíos de equilibrio, coordinación, entrenamiento de fuerza y resistencia aeróbica, ya que intervenciones de este tipo, donde se incluyen el entrenamiento cognitivo y el físico, junto con la modificación de factores de riesgo asociados al estilo de vida, han demostrado tener un impacto positivo en la prevención y disminución de caídas, debido al aumento de fuerza, movilidad, resistencia, estabilidad y balance; mejora del desempeño cognitivo global y de las actividades de la vida diaria, así como en el estado de ánimo. ${ }^{15,16,17,18}$

Durante la intervención se destinaron días de capacitación al personal de la institución (cuidadores, enfermeros, practicantes) y familiares con la finalidad de que esta fuera llevada a casa. Las sesiones incluyeron justificación teórica y una explicación detallada del proceso, concluyendo con una práctica con los usuarios de la institución.

\section{Desarrollo de protocolos de intervención para cada sentido}

Cada sentido se trabajó de la siguiente manera:

Para el sentido de la vista se creó una biblioteca digital con imágenes clasificadas en básicas y avanzadas. El objetivo se centró en que el usuario fuera capaz de identificar, localizar y dilucidar la naturaleza de los objetos en un espacio tridimensional, generar respuestas ante estímulos visuales, promover la atención, concentración de hechos, acontecimientos y favorecer las relaciones sociales.

Respecto al sentido del tacto, es esencial que los objetos utilizados se conformen de diferentes características; atendiendo a forma, textura, dureza y temperatura. Buscando lograr la identificación de los objetos que le rodeaban, así como la fomentación de habilidades manipulativas y de coordinación visomanual.

Con relación al olfato, se utilizaron elementos que ayudaron a crear un lugar idóneo, por ejemplo, difusores de aromas para aceites esenciales, fragancias o perfumes de ambiente. Se tomó en cuenta la ventilación del área para evitar la concentración de estos y que no se prestara a la confusión. Esto con la finalidad de desarrollar el sentido del olfato, discriminar olores y que el usuario logrará relacionarlos con personas, objetos y acontecimientos de su vida.

Para el sentido de la audición, se reprodujeron sonidos familiares con el objetivo de provocar y mantener el mayor tiempo posible todas aquellas habilidades auditivas residuales, la atención, localización, asociación espacial y la 
memoria auditiva. Sonidos de animales, medios de transporte, de la naturaleza, instrumentos musicales y canciones fueron los seleccionados.

Referente al sentido del gusto, se consideró mínimo un alimento con los sabores básicos: amargo, dulce, salado, ácido y umami; con el objetivo de percibir las diferencias e identificar sabores y alimentos.

El esquema corporal se decidió trabajar a través de un espejo, dibujos, rompecabezas o láminas que mostraran los segmentos corporales para establecer relaciones espaciales, identificar conceptos tridimensionales (alto, ancho y largo), la identificación guiada de segmentos individuales y pares, así como la percepción y control del propio cuerpo.

Finalmente, se registraron los hallazgos y comportamientos en formatos de seguimiento sensorial que sirvieron como herramientas para el análisis cualitativo de los resultados a manera de retroalimentación.

\section{RESULTADOS}

Del total de la población de la institución, 20 usuarios concluyeron el programa, la media de edad fue de $78.8 \pm 9.9$ años con una edad mínima de 61 años y máxima de 94 años. El 75\% (15) pertenecía al género femenino y el 15\% (5) al masculino. El trastorno neurocognitivo más frecuente fue trastorno neurocognitivo mayor por enfermedad de Alzheimer con un 55\% (11), seguido del trastorno neurocognitivo menor $35 \%$ (7) y, por último, trastorno neurocognitivo vascular mayor $10 \%$ (2).

La primera escala aplicada fue GDS, la cual determinó el estadio de DC al inicio y final del programa. El 85\% (17) de los usuarios no presentó deterioro ni mejora en su evaluación final, y el resto empeoró su situación clínica; el 5\% (1) del estadio GDS 4 y 10\% (2) del GDS 5 migraron a un estadio GDS 6 de acuerdo con las evaluaciones realizadas. Al no mostrar mejoría, la media se mantuvo en un estadio 5, es decir, con un DC de moderado a grave.

Referente a la escala NPI para evaluar la frecuencia por gravedad en las alteraciones conductuales en el usuario, se observó que un 90\% (18) presentó mejoría; $5 \%$ (1) presentó un agravamiento y el 5\% (1) restante mantuvo el mismo resultado entre la evaluación inicial y la final.

En la Figura 1 se muestra la primera evaluación con una media de $25.7 \pm$ 18.8, mientras que en la segunda evaluación fue de $16.2 \pm 13.5$. Como se puede observar hubo una desviación en la segunda evaluación, al menos el $75 \%$ se redujo, siendo esta diferencia estadísticamente significativa con un valor de $\mathrm{z}=-3.7,(\mathrm{p}=$ $0.00)$. 


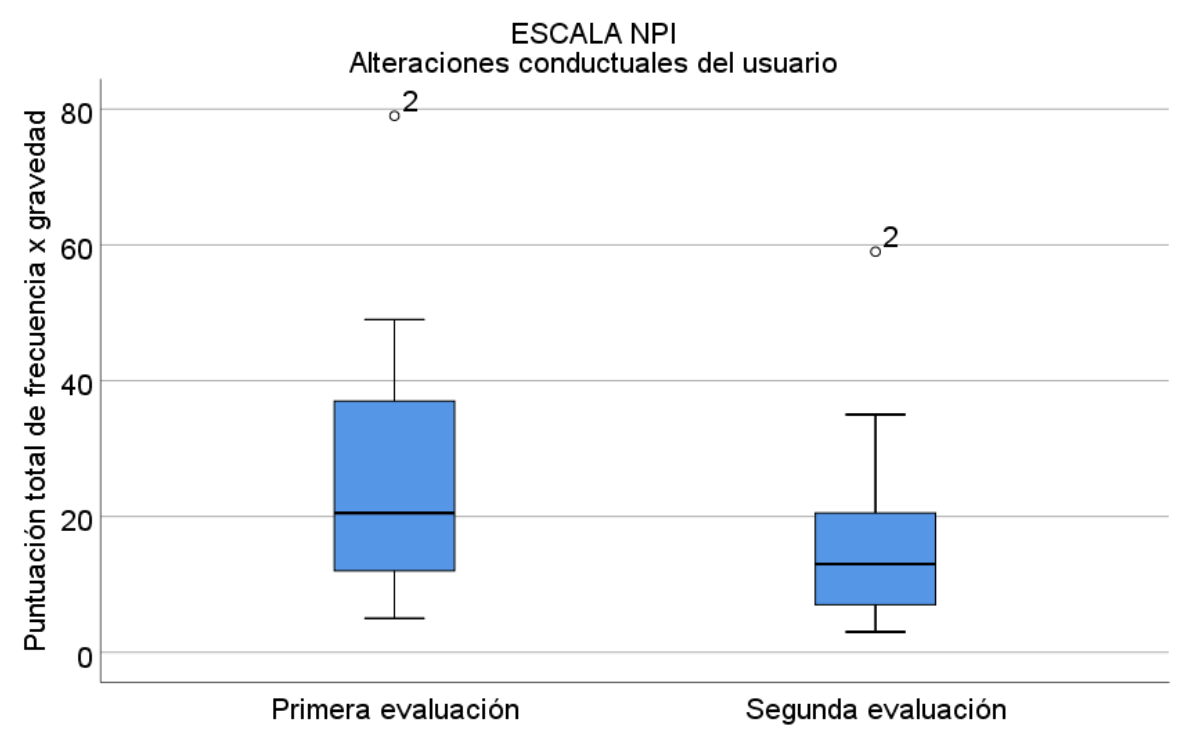

Figura 1. Comparación de las alteraciones conductuales en el usuario de acuerdo con el NPI.

En la Figura 2 se observan los cambios en el puntaje de frecuencia y gravedad de las alteraciones conductuales en cada usuario.

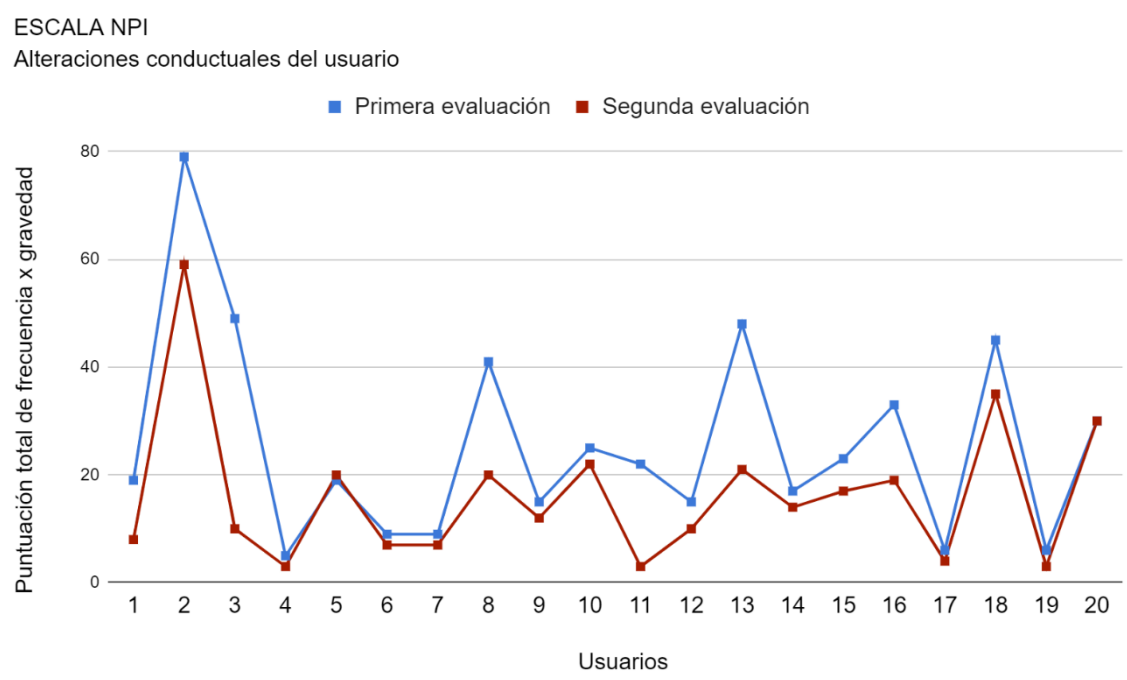

Figura 2. Puntuación de las alteraciones conductuales de cada usuario de acuerdo con el NPI antes y después de la intervención

Referente al distrés del cuidador, el 50\% (10) permaneció con el mismo puntaje, en el 40\% (8) se observó una mejoría, y solo el 10\% (2) se agravó. En la Figura 3 se puede observar una media de $14.5 \pm 6.1$ en la primera evaluación mientras que la segunda es de $10.9 \pm 2.0$. Arrojando una desviación en la segunda evaluación, con una diferencia estadísticamente significativa con un valor de $\mathrm{z}=$ $-2.5,(\mathrm{p}=0.01)$. 


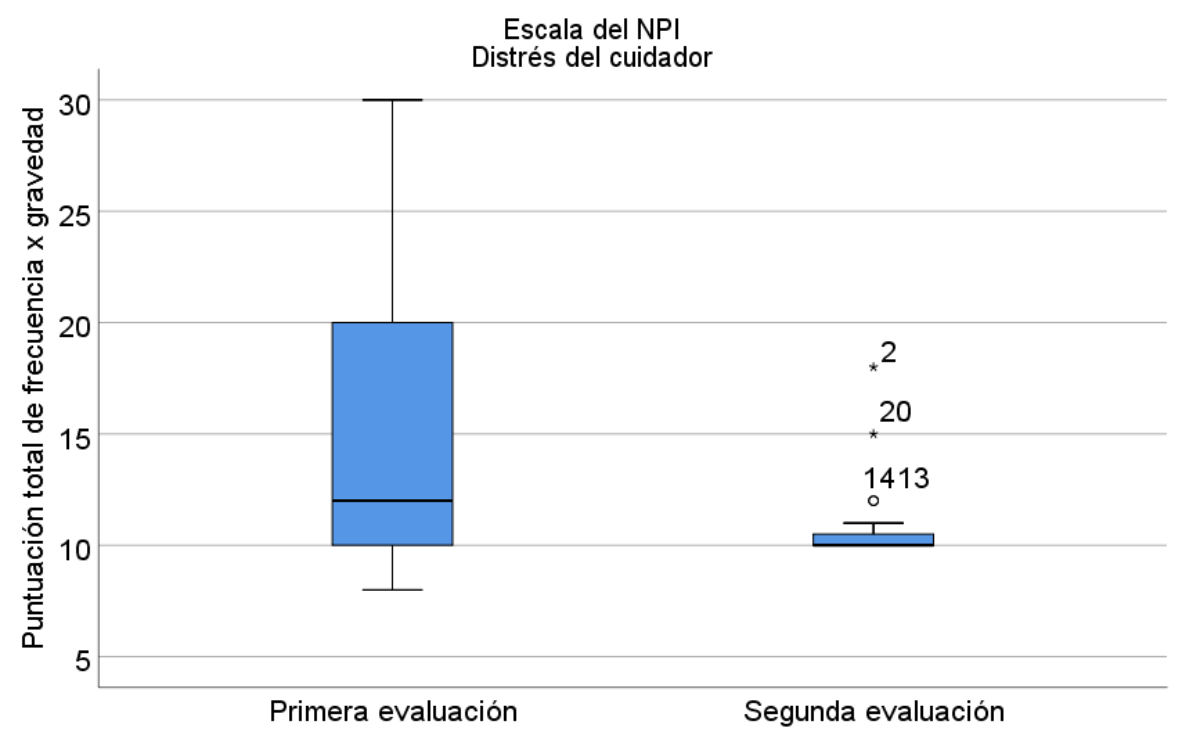

Figura 3. Comparación del distrés del cuidador primario de acuerdo con el NPI.

\section{DISCUSIÓN}

Actualmente, la evidencia que sustenta la efectividad de las intervenciones terapéuticas dirigidas a personas mayores sobre el cambio o mantenimiento del estado cognitivo, la disminución de las alteraciones conductuales o la mejora de la calidad de vida es limitada. En el presente artículo pudo observarse que la aplicación del programa de EM no tuvo un impacto sobre la mejora del grado de DC. Sin embargo, fue posible identificar una permanencia del estadio de DC en el $85 \%$ (17) de la población. Vaca y cols., ${ }^{19}$ en su estudio con pacientes que presentaban DC de leve a moderado, demostraron que la aplicación de la EM ayudó a disminuir las manifestaciones de síntomas psicológicos y conductuales, considerándose una gran alternativa terapéutica.

De igual forma, el programa de EM implementado en esta investigación tuvo efectos positivos en la disminución de las alteraciones conductuales y en el distrés del cuidador.

El proveer cuidados a una persona con trastorno neurocognitivo y alteraciones conductuales puede causar un estrés significativo, en particular sobre la persona que funge como cuidador primario. Los autores del estudio concluyen que la presencia de síntomas neuropsiquiátricos en los ancianos está relacionada con un aumento de la angustia en los cuidadores. ${ }^{20}$ Esto justifica el hecho de que al disminuir y controlar las alteraciones conductuales del usuario se disminuya el distrés del cuidador, como lo muestran nuestros resultados.

Los usuarios que participaron en el programa EM manifestaban diversas alteraciones conductuales que obstaculizaban la intervención fisioterapéutica por sí misma. Durante la aplicación del programa, se observó una disminución de estas; favoreciendo el abordaje fisioterapéutico, creando una terapia más dinámica y participativa y, a su vez, propiciando la interacción social entre usuarios. En lo que respecta al cuidador primario, se refirió una disminución en el distrés, lo que repercutió directamente en la calidad de la atención, facilitando 
las actividades de asistencia que se realizaban dentro de la institución; tales como, el manejo del usuario durante el proceso de alimentación e higiene personal, desplazamiento dentro de las áreas y la ejecución de actividades ocupacionales impartidas.

Los resultados de este estudio coinciden con los descritos por Sarabia y cols., ${ }^{21}$ quienes emplearon un protocolo de intervención sensorial similar a este, con la diferencia de que se realizó una tercera evaluación de seguimiento dos meses después de la aplicación del programa y en su metodología incluyeron un grupo control y uno experimental, demostrando una mejora estadísticamente significativa en las alteraciones conductuales post-intervención; sin embargo, no se tomó en cuenta el impacto que estos resultados representan en el distrés del cuidador.

Otro punto a destacar es la importancia de contar con una metodología bien estructurada e individualizada, ya que en la actualidad existen múltiples intervenciones terapéuticas propuestas para el manejo integral del paciente con trastorno neurocognitivo; sin embargo, suelen aplicarse de manera global y con sustentos metodológicos pobres en evidencia científica. En un estudio se aplicó un programa donde incluían actividades cognitivas y de terapia ocupacional de forma grupal; aun con la heterogeneidad de las intervenciones utilizadas y con la manera en la que se aplicó el estudio, mostró resultados sobre la reducción de los síntomas depresivos en los pacientes y en la angustia de los cuidadores. ${ }^{22}$ Sin embargo, no es posible distinguir cuál de las intervenciones es la que tuvo efecto en la modificación de las variables estudiadas, lo que refuerza la idea de que una intervención individualizada podría reflejar mejores resultados como los presentados en este estudio.

\section{CONCLUSIONES}

El trastorno neurocognitivo mayor y menor han sido declarados una prioridad global en materia de salud pública. Tras una profunda revisión de la literatura, no cabe duda de la extensa heterogeneidad de intervenciones existentes hoy día; así, nuestro estudio no es la excepción. A diferencia de estas, nuestro programa se basa en los principios de contar con una metodología sólida, dosificada de manera individual basada en las preferencias sensoriales de cada usuario tomando en cuenta sus características biopsicosociales; además de incluir a los familiares en el cuidado activo del usuario para lograr el empoderamiento, inclusión y educación de la familia. Esta integración mostró un efecto positivo sobre la situación clínica general que experimentaba el usuario; disminuyendo las alteraciones conductuales y el distrés del cuidador primario, así como facilitando la intervención fisioterapéutica. Dicho lo anterior, se sugiere que el programa forme parte del tratamiento integral del usuario.

Además de los beneficios observados en el usuario y en el cuidador, el contar con una metodología bien estructurada facilita la replicación del programa de estimulación multisensorial en instituciones dedicadas al servicio de salud y de asistencia social para la persona mayor. Por lo tanto, la EM es una alternativa de tratamiento no farmacológico que desempeña un rol importante en el manejo de los trastornos neurocognitivos, mostrando efectividad en el manejo de los aspectos conductuales, cognitivos y funcionales de los usuarios. Sin embargo 
es necesario desarrollar futuros estudios clínicos aleatorizados empleando la metodología propuesta en el presente estudio, con un seguimiento clínico a largo plazo y aumentando el tamaño de la muestra, incluyendo otras evaluaciones neuropsicológicas para obtener un nivel mayor de conocimiento sobre los efectos en distintos dominios cognitivos.

\section{Referencias}

1. Benavides C. Deterioro Cognitivo en el Adulto Mayor. Rev Mex Anest. 2017;40(2):107-12.

2. Vallejo Sánchez JM, Rodríguez Palma M. Prevalencia del deterioro cognitivo leve en mayores institucionalizados. Gerokomos. 2010;21(4):153-7.

3. Custodio N, Montesinos R, Alarcón JO. Evolución histórica del concepto y criterios actuales para el diagnóstico de demencia. Rev Neuropsiquiatr. 2019;81(4):235-249.

4. Cancino M, Rehbein L. Factores de riesgo y precursores del Deterioro Cognitivo Leve (DCL): Una mirada sinóptica. Ter Psicol. 2016;34(3):183-9.

5. Alzheimer's Disease International. Informe Mundial sobre el Alzheimer 2019. Actitudes hacia la demencia. [Internet]. Londres: Alzheimer's Disease International; 2019 p. 2112-17. Disponible en: https://www.alzint.org/u/ WorldAlzheimerReport

6. Eggert J, Dye CJ, Vincent E, Parker V, Daily SB, Pham H et al. Effects of viewing a preferred nature image and hearing preferred music on engagement, agitation, and mental status in persons with dementia. SAGE Open Medicine. 2015;3(3):1-8.

7. García Meilán JJ, Carro Ramos J. Programa de actuación cognitiva integral en demencias (PACID). [Internet]. 2011. Disponible en: https://crealzheimer.imserso.es/InterPresent2/groups/imserso/documents/ binario/guia_pacid_web.pdf

8. Castellón Sánchez del Pino A, Gómez Arques MA, Martos Martín A. Alteraciones conductuales en la enfermedad de Alzheimer. Medicina de Familia. Medicina de Familia SEMERGEN. 2005;31(11):541-545.

9. Organización Mundial de la Salud, Alzheimer's Disease International. Demencia: una prioridad de salud pública. [Internet]. Washington, D.C. 2013;1-102. Disponible en: https://www.who.int/mental_health/publications/dementia_report_2012/ es/

10. Fleming R, Zeisel J, Bennett K. World Alzheimer Report 2020: Design Dignity Dementia: dementia-related design and the built environment [Internet]. London, England: Alzheimer's Disease International; 2020 [Consultado 22 Jul 2021]. Disponible en: https://www.alzint.org/u/ WorldAlzheimerReport2020Vol1.pdf

11. Achterberg I. WorldwideSnoezelen-History (Eng version) [Internet]. Worldwidesnoezelen.nl. WorldwideSnoezelen; 2011 [Consultado 22 Jul 2021]. Disponible en: https://worldwidesnoezelen.nl/en/about-us/item/588-history-eng-version.

12. Huertas Hoyas E. La sala snoezelen en terapia ocupacional. TOG (A Coruña). 2009;6(10):1-9.

13. Reisberg B, Ferris S H, De Leon MJ, Crook T. The Global Deterioration Scale for Assessment of Primary Degenerative Dementia. Am J Psychiatry. 1982;139(9): 1136-9. 
14. Cummings JL. The Neuropsychiatric Inventory: Assessing psychopathology in dementia patients. Neurology. 1997;48(5 Suppl 6):S10-6.

15. Cui M, Lin Y, Sheng J, Zhang, X, Cui R. Exercise Intervention Associated with Cognitive Improvement in Alzheimer's Disease. Neural Plasticity. 2018:9234105.

16. Kivipelto M, Mangialasche F, Ngandu T. Lifestyle interventions to prevent cognitive impairment, dementia and Alzheimer disease. Nat Rev Neurol. 2018;14 (11):653-666.

17. Lam F, Huang M, Liao L, Chung R, Kwok T, Pang M. Physical exercise improves strength, balance, mobility, and endurance in people with cognitive impairment and dementia: a systematic review. Journal of Physiotherapy. 2018;64(1):4-15.

18. Karssemeijer E, Aaronson J, Bossers W, Smits T, Olde M, Kessels, R. Positive effects of combined cognitive and physical exercise training on cognitive function in older adults with mild cognitive impairment or dementia: A meta-analysis. Ageing Research Reviews. 2017;40:75-83.

19. Vaca R, Aznar A, Martínez P, Villa E, Espluga S, Pozo A, Ancizu I. Estimulación multisensorial en centros residenciales: Una terapia no farmacológica que mejora la conexión con el entorno. Revista de Psicogeriatría. 2019;9(1):11-17.

20. Storti L, Quintino D, Silva N, Kusumota L, Marques S. Neuropsychiatric symptoms of the elderly with Alzheimer's disease and the family caregivers' distress. Rev Lat Am Enfermagem. 2016;24:e2751

21. Sarabia CMS, Saiz ZD, Vejo CA. La estimulación multisensorial como puente de comunicación con el paciente con demencia. Nuber Científ. 2017;3(21):11-2.

22. Viola L, Nunes P, Yassuda M, Aprahamian I, Santos F, Santos G, Forlenza O. Effects of a multidisciplinar cognitive rehabilitation program for patients with mild Alzheimer's disease. Clinics. 2011;66(8):1395-1400. 